\title{
TITLE:
}

\section{Improved phase diagram of polyelectrolyte solutions}

$\operatorname{AUTHOR}(S)$ :

Nishida, K; Kaji, K; Kanaya, T

CITATION:

Nishida, K ... [et al]. Improved phase diagram of polyelectrolyte solutions. JOURNAL OF CHEMICAL PHYSICS 2001, 115(17): 8217-8220

\section{ISSUE DATE:}

2001-11-01

URL:

http://hdl.handle.net/2433/39734

\section{RIGHT:}

Copyright 2001 American Institute of Physics. This article may be downloaded for personal use only. Any other use requires prior permission of the author and the American Institute of Physics. 


\title{
Improved phase diagram of polyelectrolyte solutions
}

\author{
K. Nishida, K. Kaji, ${ }^{\text {a) }}$ and T. Kanaya \\ Institute for Chemical Research, Kyoto University, Uji, Kyoto-fu 611-0011, Japan
}

(Received 2 May 2001; accepted 10 August 2001)

\begin{abstract}
A previously reported phase diagram of polyelectrolyte solutions as functions of degree of polymerization and the concentration is improved. The new phase diagram is also intended for salt-free solutions of polyelectrolytes with the constitutional repeating unit $-\mathrm{C}-\mathrm{CX}-, \mathrm{X}$ being a charged group. The main improved point is the crossover concentrations between the dilute and semidilute regimes in the low concentration range of less than $10^{-2} \mathrm{~mol} / \mathrm{l}$, which is based on the recent most reliable experimental data on the persistence length of flexible polyion chains. This phase diagram as well introduces both the concentrated regime, which has been not only theoretically predicted but also experimentally confirmed, and the experimentally newly found swollen regime. Furthermore, it is proposed that the dilute regime is separated into four regions, gaslike, poor order, liquidlike order and crystal. The degrees of orderliness in these regions have been assumed to depend on the ratio $\delta / \xi_{\text {cent }}$ of the amplitude of thermal fluctuations to the mean intermolecular distance. Typically, $\delta / \xi_{\text {cent }}=1 / 5$ as the critical Lindemann ratio of melting is assigned for the crossover between the liquidlike order and crystal regions. Interestingly, the most probable region for the formation of a 3-D lattice is located within the experimental approach. (C) 2001 American Institute of Physics. [DOI: 10.1063/1.1408296]
\end{abstract}

\section{INTRODUCTION}

In 1988 , we proposed a phase diagram ${ }^{1}$ of aqueous saltfree solutions of polyelectrolytes as functions of concentration $C$ and contour length $L$ (degree of polymerization $N$ ). This phase diagram was drawn for polyelectrolytes of $(-\mathrm{C}-\mathrm{CX}-)_{\mathrm{N}}$ type, which we tentatively call vinyl-type polyelectrolytes in this paper, using the observed data ${ }^{1,2}$ for an experimentally accessible region as well as referring to some theories $^{3-5}$ of those days for an experimentally inaccessible region. It showed the dilute-semidilute crossover line and some interesting regimes. The crossover line was experimentally confirmed in a concentration range of $10^{-2}-1 \mathrm{~mol} / \mathrm{l}$ using the so-called characteristic peak of small-angle x-ray scattering (SAXS) for polyelectrolyte solutions. Thereafter, the light-scattering data supported the phase diagram in a considerably low-concentration region. ${ }^{6,7}$ However, below $10^{-2} \mathrm{~mol} / \mathrm{l}$ the crossover line remains questionable. Accumulated results ${ }^{8-10}$ for these ten years have made it confident that flexible polyelectrolyte chains cannot extend completely, contradicting the previous assumption. Degiorgio et al. ${ }^{8}$ examined the electrostatic persistence length $b_{\mathrm{e}}$ at very low ionic strength $I$, using transient electric birefringence and showed a tendency of the leveling-off behavior of $b_{\mathrm{e}}$ at around $I=10^{-4} \mathrm{~mol} / \mathrm{l}$. We have also extensively studied the persistence length of polyion by viscosity and small-angle neutron-scattering measurements, ${ }^{10,11}$ because the persistence length is one of the most important characteristic lengths which determines the structure of polyelectrolyte solutions. As the ultimate value of $b_{\mathrm{e}}$ for a vinyl-type polyion in the infinite dilution, we have obtained a length of the order of $500 \AA$, and proposed an empirical equation for $b_{\mathrm{e}}$ versus $I$, which is applicable to all ionic strength regions, ${ }^{10}$ based on

${ }^{a)}$ Author to whom correspondence should be addressed. the master curve of several experimental data including not only our data, but also the data reported in some literature. , $^{2,810-12}$

The finding of this empirical relation has inspired us to revise the phase diagram. The dilute-semidilute crossover line is little affected by such revision for the higher concentrations $C>10^{-2} \mathrm{~mol} / \mathrm{l}$ or the lower degrees of polymerization $N<$ (several hundreds). However, it is considerably affected at the lower concentrations or the higher degrees of polymerization. Some newly found crossover lines other than the dilute-to-semidilute crossover are also incorporated into the revised phase diagram; they are semidilute-toconcentrated and concentrated-to-swollen crossovers. In regard to the structure of the regimes distinguished by these crossovers, especially of the dilute regime, we discuss the orderliness of solutions in detail in terms of the intermolecular electrostatic potential.

Strictly speaking, our improved phase diagram has been constructed for sodium poly(styrene sulfonate) (NaPSS), but it is more general for vinyl-type polyelectrolytes except for the values of high-concentration crossovers. The quantitative application limits of this phase diagram will be remarked on in the last section. In this paper we intend to depict general characteristics of vinyl-type polyelectrolyte solutions in the whole concentration range.

\section{CROSSOVER CONCENTRATIONS}

\section{A. Dilute-to-semidilute crossover, $C^{*}$}

The dilute-to-semidilute crossover concentrations have been estimated by scattering experiments; ${ }^{1}$ they were determined from the intersections $C_{\text {scatt }}^{*}$ between two relations, $q_{\max } \sim(C / N)^{1 / 3}$ for the dilute regime and $q_{\max } \sim C^{1 / 2}$ for the semidilute regime where $q_{\max }$ is the length of scattering vector at the maximum intensity of the characteristic peak, $C$ 
and $N$ being concentration and degree of polymerization, respectively. As will be shown later, the $C_{\text {scatt }}^{*}$ does not generally agree with the real crossover concentration $C^{*}$ because the latter depends on the size of polyions, i.e., their radius of gyration $R_{g}$, but the former doesn't always depend on it. Experimentally, $C_{\text {scatt }}^{*}$ becomes lower than the actual crossover concentration $C^{*}$ with increasing $N$, and this effect is especially important for $N>$ (several hundreds). The actual dilute-to-semidilute crossover concentration is defined as a radius-of-gyration contact concentration of polyions, namely the concentration at which the mean inter-center-of-mass distance $\xi_{\text {cent }}$ equals two times as large as the radius of gyration $R_{g}$ :

$$
\xi_{\text {cent }}=2 R_{g} \text {. }
$$

The mean-square radius of gyration is given by a wormlike chain model ${ }^{13}$ as follows:

$$
\begin{aligned}
\left\langle R_{g}^{2}\right\rangle= & b_{\mathrm{t}}^{2}\left\{\left(L / 3 b_{\mathrm{t}}\right)-1+2\left(L / b_{\mathrm{t}}\right)^{-2}\left[L / b_{\mathrm{t}}-1\right.\right. \\
& \left.\left.+\exp \left(-L / b_{\mathrm{t}}\right)\right]\right\} .
\end{aligned}
$$

Here $L$ is the contour length, which is $2.5 \mathrm{~N}$ in $\AA$ for vinyltype chains and $b_{\mathrm{t}}$ is the total persistence length which is assumed $b_{\mathrm{t}}=b_{0}+b_{\mathrm{e}}, b_{0}$ being the bare persistence length. The empirical equation for the electrostatic persistence length $b_{\mathrm{e}}$ is obtained by subtracting $b_{0}$ from the observed values of the persistence length $b_{t}$, i.e., ${ }^{10}$

$$
b_{\mathrm{e}}=7.5\left(I+2 \times 10^{-4}\right)^{-1 / 2},
$$

or

$$
b_{\mathrm{e}}=7.5\left[(0.35 / 2) C+2 \times 10^{-4}\right]^{-1 / 2},
$$

where $b_{\mathrm{e}}, I$ and $C$ are given in $\AA$, mol/l and monomer mol/l, respectively. Equation (3) is obtained as a master curve of several kinds of experimental data by means of intrinsic viscosity, ${ }^{10,12}$ small-angle neutron scattering ${ }^{2,11}$ and transient electric birefringence. ${ }^{8}$ As is seen from Eq. (3), $b_{\mathrm{e}}$ is generally proportional to the reciprocal of square root of the total ionic strength $I$ of the solution. However, when $I$ or the concentration without added salt is extremely low, the localized ionic strength around the polyions becomes effective. The coefficient 7.5 and the localized ionic strength 2 $\times 10^{-4} \mathrm{~mol} / \mathrm{l}$ were determined so as to optimally well reproduce the general feature of the $I$ dependence of $b_{\mathrm{e}}$ in a wide $I$ range. Therefore, some adjustment would be required if the fine feature of $b_{\mathrm{e}}$ in a narrow $I$ range is discussed. When Eqs. (3) or (4) are substituted into Eq. (2), the value of the bare persistence length $b_{0}(=9 \sim 12 \AA),{ }^{14,2}$ which had been subtracted from the experimental value of $b_{t}$, is added again. The concentration dependencies of the empirical mean intercenter-of-mass distance $\xi_{\text {cent }}$ and intersegmental distance $\xi_{\text {seg }}$ are given as follows, respectively: ${ }^{1}$

$$
\begin{aligned}
& \xi_{\text {cent }}=(2 \pi / 0.53)(C / N)^{-1 / 3}, \\
& \xi_{\text {seg }}=(2 \pi / 0.17) C^{-1 / 2},
\end{aligned}
$$

where $\xi_{\text {cent }}, \xi_{\text {seg }}$ and $C$ are also given in $\AA$ and monomer $\mathrm{mol} / \mathrm{l}$, respectively. Figures 1 (a) and 1(b) show the concentration dependence of $2 R_{\mathrm{g}}, \xi_{\text {cent }}$ and $\xi_{\text {seg }}$ for $N=10^{2}$ and $10^{4}$, respectively. As is seen from these figures, $C_{\text {scatt }}^{*}$ and $C^{*}$

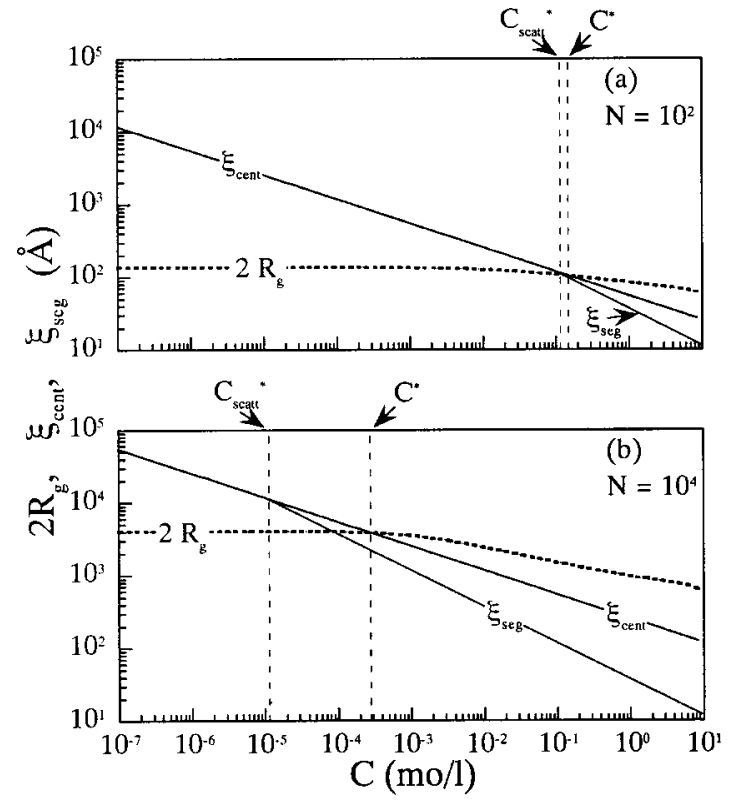

FIG. 1. Concentration dependence of two times as large as the radius of gyration $2 R_{\mathrm{g}}$, mean inter-center-of-mass distance $\xi_{\text {cent }}$ and intersegmental distance $\xi_{\text {seg }}$ for $N=10^{2}$ (a) and $10^{4}$ (b), respectively.

are very close to each other when $N$ is small, while $C_{\text {scatt }}^{*}$ appears at a considerably lower concentration than the actual $C^{*}$ when $N$ is large. The $C^{*}$ curve obtained in this way is drawn by a thick solid line in the new phase diagram of Fig. 3.

\section{B. A problem of liquid-crystalline phase in the semidilute regime}

In the previously reported phase diagram, a liquidcrystalline lattice region was tentatively introduced in the semidilute regime according to Odjik. ${ }^{5}$ The necessary (but not sufficient) condition for its appearance was very severe as follows:

$$
\xi_{\text {seg }}<L<b_{\text {t }} .
$$

As was described in the introduction, the observed $b_{\mathrm{t}}$ does not attain infinity but levels off with dilution, contradicting the theory ${ }^{4}$ adopted in the previous phase diagram. There-

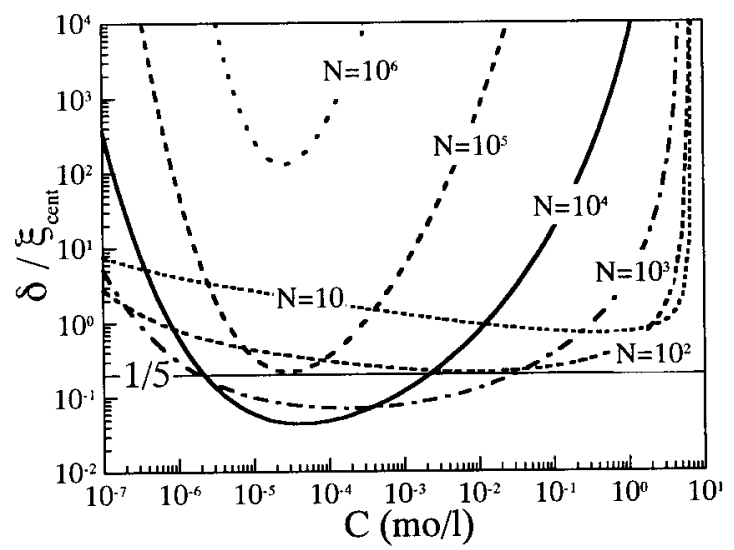

FIG. 2. Concentration dependence of the ratio of the amplitude of the thermal fluctuations to the mean inter-center-of-mass distance, $\delta / \xi_{\text {cent }}$ for some typical degrees of polymerization $N$. 


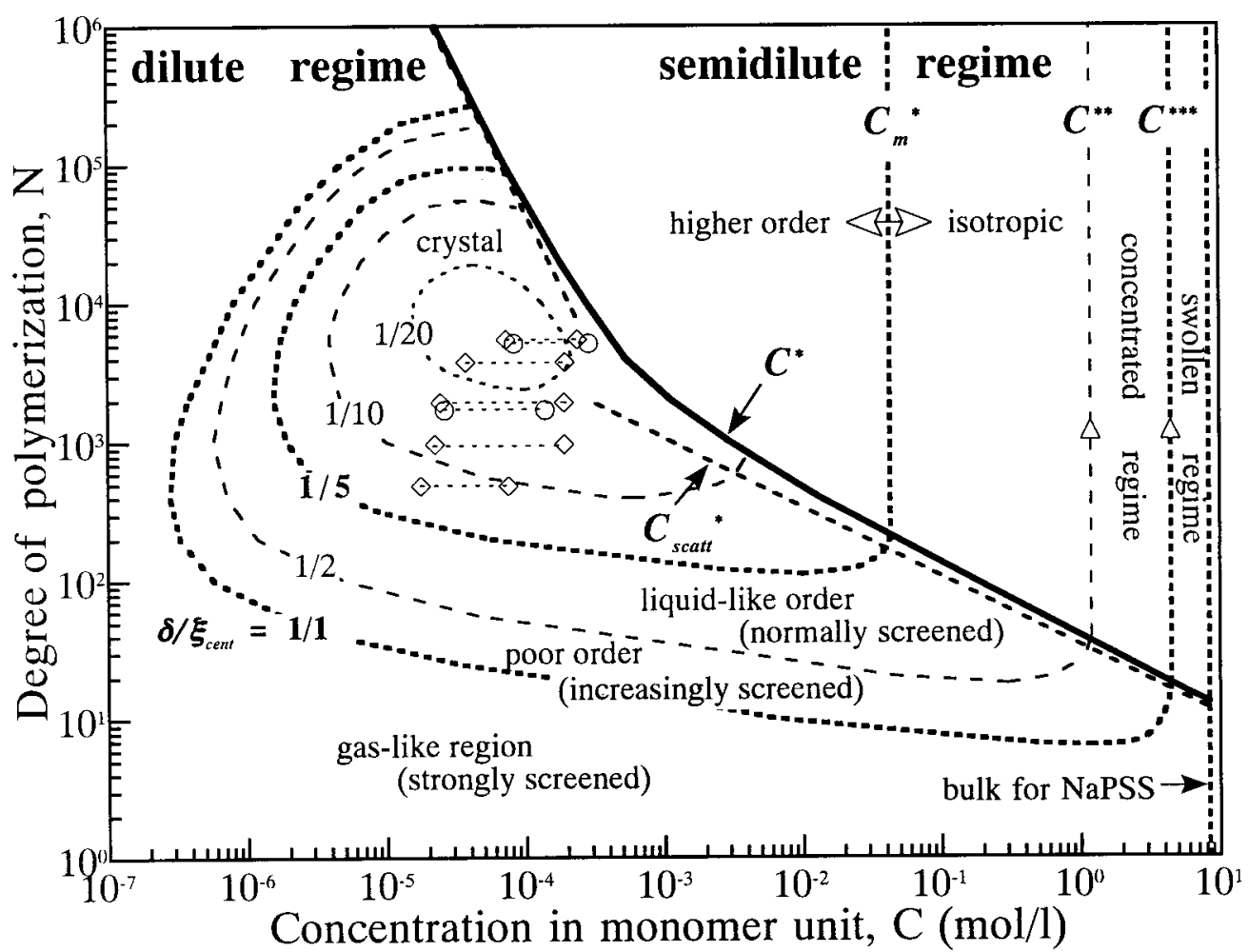

FIG. 3. The phase diagram of vinyl-type polyelectrolyte solutions as functions of degree of polymerization $N$ and the concentration $C$ in monomer mol/l. Static light-scattering data by Krause et al. ${ }^{6}$ and Johner et al. ${ }^{7}$ all of which approximately show $q_{\max } \sim(C / N)^{1 / 3}$ behavior, are marked with $(\bigcirc)$ and $(\diamond)$, respectively. The experimental points ${ }^{14}$ for the semidilute to concentrated crossover $C^{* *}$ and the concentrated to swollen crossover $C^{* * *}$ for NaPSS are marked with $(\Delta)$. Quantitative values of $C^{* *}$ and $C^{* * *}$ might change depending on the kind of polyelectrolytes or their individual molecular structure.

fore, it is now clear that the condition of Eq. (7) is never realized and we can understand why the observation of the liquid-crystalline phase has not so far been reported. Even if Eq. (7) were satisfied, the appearance of the oriented phase would be unfavorable, since for the repulsing rods the perpendicular configuration is preferred ${ }^{15}$ rather than the parallel configuration.

\section{Estimation of the degree of orderliness in the dilute regime}

The degree of orderliness in the dilute regime may be estimated from the ratio of the amplitude $\delta$ of thermal fluctuations to the mean inter-center-of-mass distance $\xi_{\text {cent }}$, based on the consideration by de Gennes et al. ${ }^{3}$ When we construct a new phase diagram, we therefore calculate the ratio $\delta / \xi_{\text {cent }}$ explicitly as functions of $N$ and $C$ assuming the Debye-type screening Coulomb potential. In the dilute regime, where the polyion is approximately taken as an $\alpha N$-valent charged point, the intermolecular potential $U(r)$ is written as follows:

$$
U(r)=\left[(\alpha N e)^{2} / 4 \pi \varepsilon r\right] \exp \left(-r / r_{\mathrm{D}}\right),
$$

where $\alpha, e, \varepsilon, r$ and $r_{\mathrm{D}}$ are the effective charge density $(=0.35$ for vinyl-type polymer $),{ }^{16,17}$ the elementary charge unit, the dielectric constant of the solvent, interparticle distance and the Debye screening length, respectively. Here, $r_{\mathrm{D}}$ is given as follows:

$$
r_{\mathrm{D}}=\left(8 \pi \ell_{\mathrm{B}} I\right)^{-1 / 2},
$$

where $I$ and $\ell_{\mathrm{B}}$ are the total ionic strength of solution and the Bjerrum length $\left(=7.2 \AA\right.$ for water at $\left.25^{\circ} \mathrm{C}\right)$, respectively. Since we are not considering an idealistic system but a realistic system, a certain value for the residual ionic strength $C_{\mathrm{r}}$ $\left(\approx 10^{-6} \mathrm{~mol} / \mathrm{l} \text { for properly deionized water }\right)^{10,18}$ should be included in the total ionic strength $I$, and hence $I$ is given as follows:

$$
I=(\alpha / 2) C+C_{\mathrm{r}} .
$$

The mean-square amplitude of the fluctuation $\delta^{2}\left(=\left\langle x^{2}\right\rangle\right)$ is given as the ratio of the thermal energy to the second derivative of $U(r):^{3}$

$$
\delta^{2}=k T /\left(\partial^{2} U / \partial r^{2}\right),
$$

and the second derivative of $U(r)$ is given by

$$
\partial^{2} U / \partial r^{2}=U(r)\left[\left(2 / r^{2}\right)+\left(2 / r_{\mathrm{D}} r\right)+\left(1 / r_{\mathrm{D}}^{2}\right)\right] .
$$

Therefore, the ratio $\delta / \xi_{\text {cent }}$ of the amplitude of thermal fluctuations to the mean inter-center-of-mass distance can be analytically calculated. Figure 2 shows the ratio $\delta / \xi_{\text {cent }}$ as a function of concentration $C$ for some typical degrees of polymerization $N$. The line $\delta / \xi_{\text {cent }}=1 / 5$ indicates that below this there exists a possibility of a latticelike structure arising according to the Lindemann law. ${ }^{19}$ If this criterion is right, a necessary condition for the latticelike structure to be formed is $N>10^{2}$, which is just as de Gennes et al. ${ }^{3}$ suggested. As seen from the figure, however, the upper limit of $N$ for such structure also exists, i.e., $N<10^{5}$. The smallest fluctuation ( $\left.\delta / \xi_{\text {cent }}\right)$ is attained at around $C=5 \times 10^{-5} \mathrm{~mol} / \mathrm{l}$ for $N=6$ 
$\times 10^{3}$. The calculated contour line corresponding to the condition of $\delta / \xi_{\text {cent }}=1 / 5$ will be indicated with a thick broken line in the new phase diagram of Fig. 3 in the next section. Another criterion $\delta / \xi_{\text {cent }}=1 / 1$ may be considered, corresponding to the crossover above which the order is completely lost, and hence the region for $\delta / \xi_{\text {cent }}>1 / 1$ is assigned to the gaslike structure (strongly screened region). In addition, the condition of $\delta / \xi_{\text {cent }}=1 / 2$ does not have a special criterion, but when the fluctuation is less than this value, the liquidlike order (normally screened region) is probably formed.

\section{PHASE DIAGRAM OF VINYL-TYPE POLYELECTROLYTE SOLUTIONS}

The crossover lines obtained by the previous discussion are drawn as functions of $N$ and $C$ in Fig. 3. Some data reported in literature $e^{6,7,14}$ are also plotted. The $C^{*}$ curve is accompanied by two asymptotic broken lines. One is the intersection $C_{\text {scatt }}^{*}$ of Eqs. (5) and (6), giving the relation $N$ $\sim C^{-1 / 2}$ in the higher concentration region, which was already confirmed in the previous paper. ${ }^{1}$ The other is the relation $N \sim C^{-2}$ in the lower concentration region. The former relation corresponds to the rod limit of the wormlike chain model of Eq. (2) with relatively short persistence length but concentration dependent $b_{\mathrm{t}}$ [Eq. (4)], while the latter corresponds to the coil limit of this model with a concentration independent $b_{\mathrm{t}}$ in spite of its being extraordinary long $b_{\mathrm{t}}$ (=540 ̊). Static light-scattering data by Krause et $a .^{6}$ and Johner et al. ${ }^{7}$ which approximately show $q_{\max } \sim(C / N)^{1 / 3}$ behavior are marked with open circles $(\bigcirc)$ and open squares $(\diamond)$ in the diagram. Some of these data locate above the extrapolated $C_{\text {scatt }}^{*}$ line, and hence they had been considered to be in the semidilute regime in the old phase diagram, ${ }^{1}$ conflicting with the observation. Now, however, all of their light-scattering data are reasonably assigned to the dilute regime in the improved phase diagram. The experimental points $^{14}$ for the semidilute-to-concentrated crossover $C^{* *}$ and the concentrated-to-swollen crossover $C^{* * *}$ are marked with triangles $(\Delta)$ in the diagram. Probably, these crossovers are independent of $N$ as long as the region above $C^{*}$ line is discussed. The $C^{* *}$ has also theoretically predicted ${ }^{20,21}$ as the semidilute-to-concentrated transition where the relation $q_{\max } \sim C^{1 / 2}$ continuously changes into $q_{\max } \sim C^{1 / 4}$ at around $C=1 \mathrm{~mol} / \mathrm{l}$. Furthermore, $C_{\mathrm{m}}^{*}$ line corresponds to the melting point of the two-dimensional lattice as expected from the Lindemann law. ${ }^{1,3,5,19}$ Interestingly, the $C^{* * *}, C^{* *}$ and $C_{\mathrm{m}}^{*}$ lines smoothly connect to the contour lines of $\delta / \xi_{\text {cent }}=1 / 1$, $1 / 2$, and $1 / 5$, respectively, on the $C^{*}$ line. Indeed, the gaslike region reasonably corresponds to the swollen regime where no structure due to electrostatic interactions is observed, ${ }^{14}$ and the liquidlike order region in the dilute regime corresponds to the isotropic regime where the intersegmental liquidlike ordering is usually observed. ${ }^{1,14,22}$ As well, the higher order region in the semidilute regime probably corresponds to the crystal region in the dilute regime. In this respect, it should be noted that higher order SAXS peaks have been observed for $C<C_{\mathrm{m}}^{*}$ in the semidilute regime according to our preliminary study.
Finally, we would like to point out that our phase diagram predicts a possibility of the "crystal" region in the dilute regime. Although such a region is not explicitly reported, the static light-scattering data corresponding to the points in the phase diagram for $N=1700(\bigcirc)$ by Krause et $a l .{ }^{6}$ and for $N=5500(\diamond)$ by Johner et al. ${ }^{7}$ showed the second Bragg peak.

\section{CONCLUDING REMARKS}

We have reconstructed the phase diagram for vinyl-type of polyelectrolyte solutions without added salt. Similar behavior is often observed among vinyl-type polyelectrolytes. Especially, both sodium poly(styrene sulfonate) (NaPSS) and sodium sulfuric acid esterificated poly(vinyl alcohol) (NaPVS) have the same type of strong acidic dissociation group, accordingly they behave almost identically ${ }^{1,22}$ and furthermore, they are compatible when $C<1 \mathrm{~mol} / \mathrm{l} .{ }^{23}$ Therefore, it may safely be said that this phase diagram is generally applicable to vinyl types of polyelectrolytes with strong acidic dissociation groups for $C<1 \mathrm{~mol} / \mathrm{l}$. However, the quantitative application for $C>1 \mathrm{~mol} / \mathrm{l}$ should be limited to NaPSS. ${ }^{14}$ We hope that this phase diagram will be useful to overlook, not only the confirmed regions, but also unconfirmed regions of such systems. In detail, some modification would be necessary depending on the individual molecular structure, especially for the intrinsic volume of the polyion. We would like to welcome both favorable and critical research.

${ }^{1}$ K. Kaji, H. Urakawa, T. Kanaya, and R. Kitamaru, J. Phys. (France) 49 993 (1988).

${ }^{2}$ M. Nierlich, F. Boué, A. Lapp, and R. Oberthür, Colloid Polym. Sci. 263, 955 (1985).

${ }^{3}$ P. G. de Gennes, P. Pincus, R. M. Velasco, and F. Brochard, J. Phys. (France) 37, 1461 (1976).

${ }^{4}$ M. Le Bret, J. Chem. Phys. 76, 6243 (1982).

${ }^{5}$ T. Odijk, Macromolecules 12, 688 (1979).

${ }^{6}$ R. Krause, E. E. Maier, M. Deggelmann, M. Hagenbüchle, S. F. Schulz, and R. Weber, Physica A 160, 135 (1989).

${ }^{7}$ C. Johner, H. Kramer, S. Batzill, C. Graf, M. Hagenbüchle, C. Martin, and R. Weber, J. Phys. II (France) 4, 1571 (1994).

${ }^{8}$ V. Degiorgio, F. Mantegazza, and R. Piazza, Europhys. Lett. 15, 75 (1991).

${ }^{9}$ S. Förster, M. Schmidt, and M. Antonietti, J. Phys. Chem. 96, 4008 (1992).

${ }^{10}$ K. Nishida, K. Kaji, T. Kanaya, and N. Fanjat, Polymer (in press).

${ }^{11}$ K. Nishida, H. Urakawa, K. Kaji, B. Gabrys, and J. S. Higgins, Polymer 38, 6083 (1997)

${ }^{12}$ M. Tricot, Macromolecules 17, 1698 (1984).

${ }^{13}$ H. Yamakawa, Modern Theory of Polymer Solutions (Harper \& Row, New York, 1971).

${ }^{14}$ K. Nishida, K. Kaji, and T. Kanaya, J. Chem. Phys. 114, 8671 (2001).

${ }^{15}$ S. L. Brenner and V. A. Parsegian, Biophys. J. 14, 327 (1974).

${ }^{16}$ F. Oosawa, J. Polym. Sci. 23, 421 (1957)

${ }^{17}$ G. S. Manning, J. Chem. Phys. 51, 924 (1969).

${ }^{18}$ H. J. Cohen, Z. Priel, and Y. Rabin, J. Chem. Phys. 88, 7111 (1988).

${ }^{19}$ See for examples, J. Ziman, Principles of the Theory of Solids (Cambridge University Press, Cambridge, 1965) Chap. 2; J. P. Hansen and J. R. McDonald, Theory of Simple Liquids (Academic, London, 1976), Chap. 10.

${ }^{20}$ J.-F. Joanny and L. Leibler, J. Phys. (France) 51, 545 (1990).

${ }^{21}$ M. Muthukumar, J. Chem. Phys. 105, 5183 (1996).

${ }^{22}$ K. Nishida, K. Kaji, and T. Kanaya, Macromolecules 28, 2472 (1995).

${ }^{23}$ K. Nishida, M. Shibata, T. Kanaya, and K. Kaji, Polymer 42, 1501 (2001). 